\title{
TOWARDS THE CAUCHY PROBLEM FOR THE LAPLACE EQUATION
}

\author{
DINH NHO HÀO, TRAN DUC VAN \\ Hanoi Institute of Mathematics \\ P.O. Box 631 Bo Ho, Hanoi, Vietnam \\ RUDOLF GORENFLO
}

FB Mathematik, Institut für Mathematik I, Freie Universität Berlin Arnimallee 2-6, D-1000 Berlin 33, Germany

1. Introduction. The Cauchy problem for elliptic equations occurs in the study of many practical problems. For example, in investigations of a gravitational (electric, magnetic) field it is often necessary to determine the potential of the field in a domain outside the mass (charge, current) creating the field, from the values of the potential in a part of this domain. A related problem is that of extending an analytic vector field in $\mathbb{R}^{3}$ or a curve in $\mathbb{R}^{2}$ to a harmonic vector field outside with determination of its singularities. Such problems play a role, e.g., in the construction of magnetohydrostatic equilibria [24, 25, 54]. The solution of this problem is equivalent to the solution of a Cauchy problem for the Laplace equation that is ill-posed in the sense of Hadamard [3, 27, 46, 81]. For an introduction to the literature of such problems the reader is referred to $[3,27$, $34,46,64,81]$.

The solution of the Cauchy problem for the Laplace equation will exist only if strong compatibility or smoothness conditions are imposed on the initial data. It was Hadamard who showed that unless a certain compatibility relation holds among the Cauchy data no global solution can exist. He further showed that even if the data are such that a classical solution exists, this solution will not depend continuously on the data $[26,27]$.

In this paper we consider the following problems $\mathrm{A}, \mathrm{B}$ and $\mathrm{C}$ :

Problem A. Find a function $u(y, x)$ which satisfies

$$
u_{y y}(y, x)+\Delta u(y, x)=0, \quad(y, x) \in \mathbb{R}_{+}^{N+1}=\left\{(y, x) \mid y>0, x \in \mathbb{R}^{N}\right\},
$$




$$
\begin{aligned}
u(0, x) & =f(x), & & x \in \mathbb{R}^{N}, \\
u_{y}(0, x) & =g(x), & & x \in \mathbb{R}^{N} .
\end{aligned}
$$

Problem B. Find a function $u(y, x)$ which satisfies the equations

$$
\begin{gathered}
u_{y y}(y, x)+u_{x x}(y, x)=0, \quad(y, x) \in D_{Y}=\{(y, x) \mid 0<y,-\pi<x<\pi\}, \\
u(0, x)=f(x), \quad u_{y}(0, x)=g(x), \quad-\pi<x<\pi .
\end{gathered}
$$

Problem C. Find a function $u(y, x)$ which satisfies

$$
\begin{gathered}
u_{y y}(y, x)+\Delta u(y, x)=0, \quad y \in(0, Y), \quad x \in(-\pi, \pi)^{N}=: \Omega, \\
u(0, x)=f(x), \quad u_{y}(0, x)=g(x), \quad x \in(-\pi, \pi)^{N}, \\
\left.u(y, x)\right|_{\partial \Omega}=0, \quad y \in(0, Y) .
\end{gathered}
$$

These problems are well known to be ill-posed in the sense of Hadamard, and many attempts, many investigations from various aspects, as existence-uniqueness theorems $[1,5,13-15,17,19,20,26,27,29-31,37,39-42,47,48,51,52$, $57-59,61,62-67,69,73-79,83,94-99,100,101,104,106]$, stability estimates [3, $4,7,8,21,33,35,38,40,43-45,48,53,55]$, regularization, least square methods $[21,28,32,48,56,81,92, \ldots]$ for such problems have been discussed.

We shall use the theory of pseudodifferential operators with real analytic symbols ( $\Psi$ DOAS) $[18,84-89]$ and the approximation theory of functions to regularize the above problems. We observe that every function in $L_{p}\left(\mathbb{R}^{N}\right)$ can be approximated by a sequence of entire functions of exponential type on $\mathbb{C}^{N}$, the restrictions of which to $\mathbb{R}^{N}$ belong to $L_{p}\left(\mathbb{R}^{N}\right)[60]$ (class $\mathfrak{M}_{\nu p}$ ), and in these classes our problems are well-posed. Therefore, we should first consider our problems with data in $\mathfrak{M}_{\nu p}$ and then approximate "improper" data in $L_{p}$ by $\mathfrak{M}_{\nu p}$ functions and suggest the solutions with these approximation data as a regularization. We call this method the mollification method. Tran Duc Van in his joint work with other authors [87] has used this idea to approximate the solution of the well-posed Cauchy problem for the wave equation. Dinh Nho Hào in his joint work with Gorenflo [16] has observed that this method with a modification still works for many ill-posed problems. We hope to present an abstract version of this method in a later work.

In Section II we give a short survey on the Cauchy problem for elliptic equations. Section III is devoted to Problems A, B and C. Numerical experiments will be described in a succeeding work.

This paper was written during the stay of the first and second authors at the Free University of Berlin (West). This research stay was supported by DAAD (German Academic Exchange Service) and by the Alexander von HumboldtStiftung. The authors are members of the research group "Regularization".

\section{A short survey}

2.1. Existence and uniqueness theorems. The first result on the Cauchy problem for the Laplace equation is that by Hadamard [26]. He proved, for a particular case of Problem B, the following 
THEOREM 2.1. A necessary and sufficient condition for the existence of a function $u(y, x)$ which satisfies $u_{y y}+u_{x x}=0$ in a neighborhood of the interval $-\pi<x<\pi$ of the $x$-axis, where $y>0$ and $u(0, x)=f(x), u_{y}(0, x)=g(x)$, $-\pi<x<\pi$, is that

$$
H(x)=f(x)-\frac{1}{\pi} \int_{-\pi}^{\pi} g(\xi) \ln |x-\xi| d \xi
$$

be an analytic function of $x$ for $-\pi<x<\pi$. Here $f$ is of class $C^{1}$ and $g$ is of class $C^{0}$ in $(-\pi, \pi)$.

An analogous result for Problem $\mathrm{C}$ has been obtained by Payne and Sather in [67]. Furthermore, these authors have generalized the result of Hadamard to a class of degenerate elliptic equations.

THEOREM 2.2. A necessary and sufficient condition for the existence of a function $u(y, x)$ which satisfies $u_{y y}+y^{\alpha} u_{x x}=0(\alpha>0)$ in a neighborhood of the interval $(-\pi, \pi)$ of the $x$-axis, where $y>0$, and $u(0, x)=f(x), u_{y}(0, x)=g(x)$, $-\pi<x<\pi$, is that

$$
H_{\alpha}(x)=f(x)+m_{\beta} \int_{-\pi}^{\pi} \frac{g(\xi)}{|x-\xi|^{1-\beta}} d \xi \quad\left(\beta=\frac{2}{\alpha+2}\right)
$$

be an analytic function of $x$ for $-\pi<x<\pi$. Here $f$ is of class $C^{1}$ and $g$ is of class $C^{0}$ in $(-\pi, \pi)$, and

$$
m_{\beta}=\frac{\beta^{1-\beta} \Gamma\left(\frac{1}{2}(3-\beta)\right)}{\sqrt{\pi}(1-\beta) \Gamma\left(\frac{1}{2}(2-\beta)\right)} .
$$

An interesting result on existence and uniqueness for Problem A has been given by B. H. Li and Y. Q. Li [49]:

TheOREM 2.3. Let $f, g \in \mathcal{D}^{\prime}\left(\mathbb{R}^{N}\right)$ and let (1.2), (1.3) be satisfied in the sense of $\mathcal{D}^{\prime}$. Then Problem A is solvable if and only if

$$
\mathcal{D}_{y \searrow 0}^{\prime}-\lim _{y} \bar{f}_{y}(y, x)-g
$$

is an entire function, where $\bar{f}$ is a harmonic function such that

$$
\mathcal{D}_{y \searrow 0}^{\prime}-\lim \bar{f}(y, x)=f(x) .
$$

In [85], as a direct consequence of the result of Tran Duc Van in his recent work on $\Psi$ DOAS [84], we have also obtained an existence and uniqueness theorem for Problem A. Before outlining this result we need some definitions.

Let $x \in \mathbb{R}_{x}^{N}, N \geq 1$, and $\xi \in \mathbb{R}_{\xi}^{N}$ be real variables, $D^{\alpha}=D_{1}^{\alpha_{1}} \ldots D_{N}^{\alpha_{N}}$, $D_{j}=-i \partial / \partial x_{j}, j=1, \ldots, N, \alpha=\left(\alpha_{1}, \ldots, \alpha_{N}\right),|\alpha|=\alpha_{1}+\ldots+\alpha_{N}$.

Definition 1. The space $W^{+\infty}\left(\mathbb{R}_{x}^{N}\right)$ is the set of functions $f: \mathbb{R}_{x}^{N} \rightarrow \mathbb{C}$, satisfying the following conditions: $f$ admits analytic continuation to an entire 
function on $\mathbb{C}^{N}$ and for each $\varepsilon>0$ there exist constants $r<\infty$ and $C_{\varepsilon}$, possibly depending on $f$, such that

$$
|f(x+i y)| \leq C_{\varepsilon} \exp (r|y|+\varepsilon|x|), \quad x+i y \in \mathbb{C}^{N} .
$$

We list here some classes of functions which belong to $W^{+\infty}\left(\mathbb{R}_{x}^{N}\right)$ : all functions $f \in L_{2}\left(\mathbb{R}_{x}^{N}\right)$ the support of whose Fourier transform $\widehat{f}(\xi)$ is compact (bandlimited functions) [18], all functions in $\mathfrak{M}_{\nu p}, 1 \leq p \leq+\infty, \nu<\infty$ [60], all functions in $W^{+\infty}\left(\mathbb{R}^{N}\right)[88,89]$. From the Paley-Wiener theorem it follows that $f \in W^{+\infty}\left(\mathbb{R}_{x}^{N}\right)$ if and only if its analytic continuation $f(z)$ is the Fourier-Laplace transform of an analytic functional with compact support.

Definition 2. A sequence of functions $f_{n} \in W^{+\infty}\left(\mathbb{R}_{x}^{N}\right)$ is said to converge to $f \in W^{+\infty}\left(\mathbb{R}_{x}^{N}\right)$ if and only if for each $\varepsilon>0$ there exists a constant $r<\infty$ such that (with $z=x+i y, x \in \mathbb{R}^{N}, y \in \mathbb{R}^{N}$ )

$$
\sup _{z \in \mathbb{C}^{N}}\left|f_{n}(z)-f(z)\right| \exp (-r|y|-\varepsilon|x|) \rightarrow 0, \quad n \rightarrow \infty .
$$

Let the entire function $A(\xi), \xi \in \mathbb{R}_{\xi}^{N}$, be expanded in the Taylor series

$$
A(\xi)=\sum_{|\alpha|=0}^{\infty} a_{\alpha} \xi^{\alpha}, \quad a_{\alpha}=(i D)^{\alpha} A(0) / \alpha !
$$

Denote by $W^{-\infty}\left(\mathbb{R}_{x}^{N}\right)$ the space of all continuous linear functionals defined on $W^{+\infty}\left(\mathbb{R}_{x}^{N}\right)$. Let $h \in W^{-\infty}\left(\mathbb{R}_{x}^{N}\right)$ and

$$
\langle A(D) h, \varphi\rangle:=\langle h, A(-D) \varphi\rangle, \quad \forall \varphi \in W^{+\infty}\left(\mathbb{R}_{x}^{N}\right) .
$$

TheOREM 2.4. Let $f, g \in W^{+\infty}\left(\mathbb{R}_{x}^{N}\right)\left(W^{-\infty}\left(\mathbb{R}_{x}^{N}\right)\right)$. Then there exists a unique solution of Problem A in $C^{2}\left(\mathbb{R}_{+}^{1} ; W^{+\infty}\left(\mathbb{R}_{x}^{N}\right)\right)\left(C^{2}\left(\mathbb{R}_{+}^{N} ; W^{-\infty}\left(\mathbb{R}_{x}^{N}\right)\right)\right.$, and

$$
u(y, x)=\sum_{n=0}^{\infty} \frac{(-1)^{n} y^{2 n} \Delta^{n} f(x)}{(2 n) !}+\sum_{n=1}^{\infty} \frac{(-1)^{n-1} y^{2 n-1} \Delta^{n-1} g(x)}{(2 n-1) !} .
$$

For any $y>0$, the right side of (2.5) converges in the sense of $W^{+\infty}\left(\mathbb{R}_{x}^{N}\right)$ $\left(W^{-\infty}\left(\mathbb{R}_{x}^{N}\right)\right)$.

Medeiros in [52] has given another existence and uniqueness theorem for Problem C.

Uniqueness results have received quite extensive treatment $[1,5,9,13-15,20$, $26,29,30,31,37,39,40-47,49,51-53,57-59,61-67,69-79,85,94-99,100-$ 102, 106]. We note here again important works by Carleman [9], Hörmander [30, 31], Lavrent'ev [43, 44], Lavrent'ev, Romanov and Shishat-skiǔ [46], Müller [59], Heinz [29], Cordes [15], Landis [40-42] and by Payne and his collaborators [37, 62-67]. Uniqueness results for elliptic equations with multiple characteristics were considered in [94-99, 106].

2.2. Stable methods for solutions. The first results concerning stable methods to solve the Cauchy problems for the Laplace equation are contained in the works 
of Lavrent'ev [43, 45] and Pucci [70, 71] (see also John [35]). These authors established stability estimates and proposed some stable methods. For further works see $[51,53]$. Other approaches have been tried, such as: establishing stability estimates $[3,4,7,8,21,22,33-35,46,53]$, regularization by various methods, e.g., least squares, Carleman estimates, stable summation of Fourier series, finding a Carleman function, Tikhonov regularization ... [6, 7, 20, 21, 22, 28, 55, 56, 81, 90-93, 104, ...]. For a function-theoretic method see $[11,12]$.

\section{A mollification method}

3.1. Problem A. Suppose that instead of the exact data $f$ and $g$ (which are supposed to belong to $\left.L_{p}\left(\mathbb{R}^{N}\right), 1 \leq p \leq \infty\right)$ we only have the measured data $f_{\varepsilon}$ and $g_{\varepsilon}$ such that

$$
\left\|f_{\varepsilon}-f\right\| \leq \varepsilon, \quad\left\|g_{\varepsilon}-g\right\| \leq \varepsilon .
$$

We introduce mollification operators $M_{\nu}$ which map a function $f \in L_{p}$ to a function $f_{\nu}$ in $\mathfrak{M}_{\nu p}$, and we require that $f_{\nu} \rightarrow f$ in $L_{p}$ as $\nu=\left(\nu_{1}, \ldots, \nu_{N}\right) \rightarrow \infty$.

For simplicity of presentation, we first describe results only for the case $N=1$.

We need some notations. Let $\Delta_{h} f=\Delta_{h} f(x)=f(x+h)-f(x)$, where $h$ is any real number. Then

$$
\begin{aligned}
\Delta_{h}^{k} f(x) & =\Delta_{h} \Delta_{h}^{k-1} f(x)=\sum_{l=0}^{k}(-1)^{l+k}\left(\begin{array}{l}
k \\
l
\end{array}\right) f(x+l h), \quad k \in\{1,2, \ldots\}, \\
\Delta_{h}^{0} & =f .
\end{aligned}
$$

Let

$$
\omega^{k}(\delta)=\omega^{k}(f, \delta)_{p}=\sup _{|h| \leq \delta}\left\|\Delta_{h}^{k} f(\cdot)\right\|_{p}, \quad \omega(\delta)=\omega(f, \delta)_{p}=\omega^{1}(f, \delta) .
$$

$\omega^{k}(\delta)$ is called the modulus of continuity of order $k$ of the function $f$ in the $L_{p}$ metric. It is well known that if $f \in L_{p}(\mathbb{R}), 1 \leq p<\infty$, then $\lim _{\delta \rightarrow 0} \omega(\delta)=0$. For $p=\infty$ this property does not hold in general. However, it is satisfied in a trivial way if $f$ is uniformly continuous on every compact subset of $\mathbb{R}$.

Suppose that $K(\xi)$ is a nonnegative even function of one variable, of exponential type 1 , satisfying the condition

$$
\int_{-\infty}^{\infty} K(t) d t=1
$$

and $K$ is chosen so that the integral

$$
\int_{-\infty}^{\infty} K(\xi)|\xi|^{l} d \xi,
$$

where $l$ is a fixed natural number, is finite. For $K$ we may choose a function of 
the form

$$
\mu\left(\frac{\sin (\xi / \lambda)}{\xi}\right)^{\lambda}
$$

where $\lambda \geq l+2$ is an even number and $\mu$ is a positive constant such that (3.2) holds.

For $f \in L_{p}(\mathbb{R})$ and $\nu \in \mathbb{R}^{+}$the function

$$
f_{\nu}(x)=M_{\nu} f(x)=\int_{-\infty}^{\infty} K_{\nu}(\xi-x) f(\xi) d \xi
$$

where

$$
K_{\nu}(\xi)=\sum_{j=1}^{l}(-1)^{j-1}\left(\begin{array}{l}
l \\
j
\end{array}\right) \frac{\nu}{j} K\left(\frac{\xi \nu}{j}\right)
$$

is well defined. Since $K$ is an entire function of one variable, of exponential type $1, f_{\nu}$ is an entire function of spherical type $\nu$, lying in $L_{p}(\mathbb{R})([60]$, p. 186). Now we have the following

LEMma 1 ([60], §5.2). If $f$ has derivatives of order $m$ lying in $L_{p}, k=l-m$, $0 \leq m \leq l$, then $f_{\nu}$ is an entire function of spherical type $\nu$ and

$$
\left\|f_{\nu}-f\right\|_{p} \leq \frac{c(m)}{\nu^{m}} \omega^{k}\left(f^{(m)}, 1 / \nu\right)_{p}
$$

where

$$
c(m)=\int_{-\infty}^{\infty} K(|\xi|)|\xi|^{m}(1+|\xi|)^{k} d \xi .
$$

If $s$ is a nonnegative integer with $s \leq m$ then

$$
\left\|f_{\nu}^{(s)}-f^{(s)}\right\|_{p} \leq \frac{c}{\nu^{m-s}} \omega^{k}\left(f^{(s)}, 1 / \nu\right)_{p}, \quad \nu>0,
$$

where

$$
c=\int_{0}^{\infty} K(\xi)(1+|\xi|)^{l} d \xi .
$$

Now instead of (1.1)-(1.3) with the measured data $f_{\varepsilon}$ and $g_{\varepsilon}$ we consider the mollified problem: Find $u_{\nu}^{\varepsilon}(y, x)$ such that

$$
\begin{aligned}
\frac{\partial^{2} u_{\nu}^{\varepsilon}(y, x)}{\partial y^{2}}+\frac{\partial^{2} u_{\nu}^{\varepsilon}(y, x)}{\partial x^{2}} & =0, & & (y, x) \in \mathbb{R}^{+} \times \mathbb{R} \\
u_{\nu}^{\varepsilon}(0, x) & =f_{\nu}^{\varepsilon}(x), & & x \in \mathbb{R}, \\
\frac{\partial u_{\nu}^{\varepsilon}(0, x)}{\partial y} & =g_{\nu}^{\varepsilon}(x), & & x \in \mathbb{R} .
\end{aligned}
$$


Theorem 3.1. Problem (3.11)-(3.13) is well-posed in $C^{2}\left(\mathbb{R} ; W^{+\infty}\left(\mathbb{R}_{x}^{N}\right)\right)$ and

$$
u_{\nu}^{\varepsilon}(y, x)=\sum_{n=0}^{\infty} \frac{(-1)^{n} y^{2 n}\left(f_{\nu}^{\varepsilon}\right)^{(2 n)}(x)}{(2 n) !}+\sum_{n=0}^{\infty} \frac{(-1)^{n-1} y^{2 n-1}\left(g_{\nu}^{\varepsilon}\right)^{(2 n-1)}(x)}{(2 n-1) !}
$$

Furthermore, for every $m, 0 \leq m \leq l, k=l-m$,

$$
\begin{aligned}
\| u_{\nu}^{\varepsilon}(y, \cdot)- & u(y, \cdot) \|_{p} \\
\leq & \frac{c(m)}{\nu^{m}} \omega^{k}\left(\frac{\partial^{m} u(y, \cdot)}{\partial x^{m}}, \frac{1}{\nu}\right)_{p}+c_{l} \varepsilon\left[\cosh (y \nu)+\frac{\sinh (y \nu)}{\nu}\right],
\end{aligned}
$$

where $c_{l} \leq 2^{l}-1$ does not depend on $\nu$.

Proof. Formula (3.14) is a consequence of Theorem 2.4. We have

$$
\begin{aligned}
u_{\nu}^{\varepsilon}(y, x)-u(y, x)= & \cos (y d / d x) f_{\nu}^{\varepsilon}+[\sin (y d / d x) /(d / d x)] g_{\nu}^{\varepsilon} \\
& -\cos (y d / d x) f+[\sin (y d / d x) /(d / d x)] g \\
=\{ & \cos (y d / d x) f_{\nu}^{\varepsilon}-\cos (y d / d x) f_{\nu} \\
& \left.+[\sin (y d / d x) /(d / d x)] g_{\nu}^{\varepsilon}-[\sin (y d / d x) /(d / d x)] g_{\nu}\right\} \\
& +\left\{\cos (y d / d x) f_{\nu}-\cos (y d / d x) f\right. \\
& \left.+[\sin (y d / d x) /(d / d x)] g_{\nu}-[\sin (y d / d x) /(d / d x)] g\right\} \\
=: & \Sigma_{1}+\Sigma_{2} .
\end{aligned}
$$

From the fact that $f_{\nu}, g_{\nu} \in \mathfrak{M}_{\nu p}$ and (3.5) it can be verified that

$$
\begin{aligned}
\cos (y d / d x) f_{\nu}+[ & \sin (y d / d x) /(d / d x)] g_{\nu} \\
& =M_{\nu}(\cos (y d / d x) f+[\sin (y d / d x) /(d / d x)] g)=M_{\nu} u(y, x) .
\end{aligned}
$$

Thus,

$$
\left\|\Sigma_{2}\right\|_{p}=\left\|M_{\nu}(u(y, \cdot))-u(y, \cdot)\right\|_{p} \leq \frac{c(m)}{\nu^{m}} \omega^{k}\left(\frac{\partial^{m} u(y, \cdot)}{\partial x^{m}}, \frac{1}{\nu}\right)_{p} .
$$

On the other hand, since $f_{\nu}^{\varepsilon}, f_{\nu}, g_{\nu}^{\varepsilon}, g_{\nu}$ belong to $\mathfrak{M}_{\nu p}$, we obtain from the Bernstein-Nikol'skiu inequalities ([60], p. 115)

$$
\left\|\Sigma_{1}\right\|_{p} \leq \cosh (y \nu)\left\|f_{\nu}^{\varepsilon}-f_{\nu}\right\|_{p}+\frac{\sinh (y \nu)}{\nu}\left\|g_{\nu}^{\varepsilon}-g_{\nu}\right\|_{p}
$$

But

$$
\begin{aligned}
\left\|f_{\nu}^{\varepsilon}-f_{\nu}\right\|_{p} & =\left\|\int_{-\infty}^{\infty} K_{\nu}(\xi-x)\left(f^{\varepsilon}(x)-f(x)\right) d x\right\|_{p} \\
& \leq \int_{-\infty}^{\infty}\left|K_{\nu}(\xi)\right| d \xi\left\|f^{\varepsilon}-f\right\|_{p} \leq c_{l} \varepsilon
\end{aligned}
$$

where $c_{l} \leq 2^{l}-1$ does not depend on $\nu$ ([60], p. 190). Analogously,

$$
\left\|g_{\nu}^{\varepsilon}-g_{\nu}\right\|_{p} \leq c_{l} \varepsilon
$$


Thus,

and (3.15) is proved.

$$
\left\|\Sigma_{1}\right\|_{p} \leq c_{l} \varepsilon\left[\cosh (y \nu)+\frac{\sinh (y \nu)}{\nu}\right],
$$

ThEOREM 3.2. Let

$$
\left\|\partial^{l} u(y, \cdot) / \partial x^{l}\right\|_{p} \leq M \quad \text { for } y \in[0, Y],
$$

where $m$ is a nonnegative integer. Then, with $m=l-1$,

$$
\left\|u_{\nu}^{\varepsilon}(y, \cdot)-u(y, \cdot)\right\|_{p} \leq \frac{c(m) M}{\nu^{l}}+2 c_{l} \varepsilon e^{y \nu} .
$$

Furthermore, for $y>0$ let

$$
\Omega(\varepsilon)=\inf _{\nu>0}\left\{\frac{c(m) M}{\nu^{l}}+2 c_{l} \varepsilon e^{y \nu}\right\} .
$$

Then

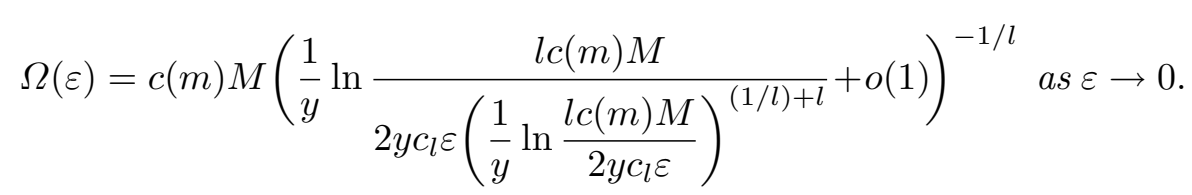

The infimum on the right hand side of (3.18) is attained if $\nu$ is the solution $\nu(\varepsilon)$ of the equation

$$
\varepsilon=\frac{c(m) M l}{2 y c_{l}} \frac{e^{-y \nu^{2}}}{\nu^{l+2}},
$$

which can be written in the form

$$
\nu(\varepsilon)=\left(\frac{1}{y} \ln \frac{l c(m) M}{2 y c_{l} \varepsilon\left(\frac{1}{y} \ln \frac{l c(m) M}{2 y c_{l} \varepsilon}\right)^{(1 / l)+l}}+o(1)\right)^{-1 / l} \quad \text { as } \varepsilon \rightarrow 0 .
$$

Proof. The estimate (3.17) is a direct consequence of (3.15) and (3.16). To prove (3.19)-(3.21) we need the following result:

LEMMA 2 ([16]). Let

$$
\Omega_{c}(\varepsilon)=\inf _{\delta>0}(\delta+c(\delta) \varepsilon) .
$$

If $c(\delta)=c_{0} \exp \left(s \delta^{-\eta}\right), \eta>0$, then

$$
\Omega_{c}(\varepsilon)=\left(\frac{1}{s} \ln \frac{1}{s c_{0} \eta \varepsilon\left(\frac{1}{s} \ln \frac{1}{s c_{0} \eta \varepsilon}\right)^{(\eta+1) / \eta}}+o(1)\right)^{-\eta} \quad \text { as } \varepsilon \rightarrow 0 .
$$

The infimum is attained if $\delta$ is the solution $\delta(\varepsilon)$ of the equation

$$
\varepsilon=\frac{1}{c_{0} s \eta} \delta^{\eta+1} e^{-s \delta^{-\eta}}
$$


which can be written in the form

$$
\delta(\varepsilon)=\left(\frac{1}{s} \ln \frac{1}{s c_{0} \eta \varepsilon\left(\frac{1}{s} \ln \frac{1}{s c_{0} \eta \varepsilon}\right)^{(\eta+1) / \eta}}+o(1)\right)^{-\eta} \quad \text { as } \varepsilon \rightarrow 0 .
$$

Our estimates (3.19)-(3.21) now follow directly from this lemma if we take $c_{0}=c_{l} / c(m) M, s=y, \eta=2 / l$.

When $N>1$, we can also approximate the measured data $f^{\varepsilon}$ and $g^{\varepsilon}$ by functions of classes $\mathfrak{M}_{\nu p}, \nu=\left(\nu_{1}, \ldots, \nu_{N}\right)$, by the above process, but since the description is somewhat lengthy and no new idea is offered, we do not write it down here.

3.2. Problem B. Instead of the exact data $f$ and $g$ in (1.5), let there be given measured data $f^{\varepsilon}$ and $g^{\varepsilon}$ such that

$$
\left\|f-f^{\varepsilon}\right\|_{L_{p}(-\pi, \pi)} \leq \varepsilon, \quad\left\|g-g^{\varepsilon}\right\|_{L_{p}(-\pi, \pi)} \leq \varepsilon .
$$

The following lemma helps us to treat this problem.

LEMMA 3. If $f$ and $g$ can be extended to entire functions in $\mathbb{R}$ of exponential type, then the solution of Problem $\mathrm{B}$ can be written in the form

$$
u(y, x)=\sum_{n=0}^{\infty} \frac{(-1)^{n} y^{2 n} f^{(2 n)}(x)}{(2 n) !}+\sum_{n=1}^{\infty} \frac{(-1)^{n-1} y^{2 n-1} g^{(2 n-1)}(x)}{(2 n-1) !} .
$$

Furthermore, this series converges in the sense of $W^{\infty}$, for fixed $y \in(0, Y)$, uniformly in $x$ for $|x|<\pi$.

Proof. Denote the extensions of $f$ and $g$ to all of $\mathbb{R}$ by the same notations $f$ and $g$, respectively. Now we consider Problem A with these data. By Theorem 2.4 the solution of Problem A exists uniquely in $W^{\infty}\left(\mathbb{R}_{x}\right)$ and

$$
u(y, x)=\sum_{n=0}^{\infty} \frac{(-1)^{n} y^{2 n} f^{(2 n)}(x)}{(2 n) !}+\sum_{n=1}^{\infty} \frac{(-1)^{n-1} y^{2 n-1} g^{(2 n-1)}(x)}{(2 n-1) !} .
$$

For fixed $y \in(0, Y)$ this series converges uniformly in every compact subset of $\mathbb{R}$.

It is clear that the function $u(y, x)$ satisfies (1.4)-(1.5). On the other hand, since $g(x)$ is an entire function of exponential type, so is $\int_{-\pi}^{\pi} g(\xi) \ln |x-\xi| d \xi$ ([60], §3.6). Thus $f(x)-\frac{1}{2 \pi} \int_{-\pi}^{\pi} g(\xi) \ln |x-\xi| d \xi$ is also an entire function, or Problem B has a unique solution (Theorem 2.1). The lemma is proved.

Now for $\nu \in \mathbb{R}^{+}$we define

$$
\begin{aligned}
& f_{\nu}^{\varepsilon}(x)=M_{\nu}^{*} f^{\varepsilon}(x)=\int_{-\pi}^{\pi} K_{\nu}(\xi-x) f^{\varepsilon}(\xi) d \xi \\
& g_{\nu}^{\varepsilon}(x)=M_{\nu}^{*} g^{\varepsilon}(x)=\int_{-\pi}^{\pi} K_{\nu}(\xi-x) g^{\varepsilon}(\xi) d \xi .
\end{aligned}
$$


By Lemma $1, f_{\nu}^{\varepsilon}$ and $g_{\nu}^{\varepsilon}$ are elements of $\mathfrak{M}_{\nu p}$. Therefore, from Lemma 3,

$$
u_{\nu}^{\varepsilon}(y, x)=\sum_{n=0}^{\infty} \frac{(-1)^{n} y^{2 n}\left(f_{\nu}^{\varepsilon}\right)^{(2 n)}(x)}{(2 n) !}+\sum_{n=1}^{\infty} \frac{(-1)^{n-1} y^{2 n-1}\left(g_{\nu}^{\varepsilon}\right)^{(2 n-1)}(x)}{(2 n-1) !}
$$

is the unique solution of Problem B with the data $f_{\nu}^{\varepsilon}$ instead of $f$, and $g_{\nu}^{\varepsilon}$ instead of $g$. We hope that $u_{\nu}^{\varepsilon}$ can serve as an approximation to $u(y, x)$. Indeed, the following theorems will show how well $u_{\nu}^{\varepsilon}(y, x)$ approximates $u(y, x)$.

TheORem 3.3. For every $m, 0 \leq m \leq l, k=l-m$, we have

$$
\begin{aligned}
\| u_{\nu}^{\varepsilon}(y, \cdot)-u(y, \cdot) & \|_{L_{p}(-\pi, \pi)} \\
& \leq \frac{c(m)}{\nu^{m}} \omega^{k}\left(\frac{\partial^{m} u(y, \cdot)}{\partial x^{m}}, \frac{1}{\nu}\right)_{p}+c_{l} \varepsilon\left[\cosh (y \nu)+\frac{\sinh (y \nu)}{\nu}\right],
\end{aligned}
$$

where $c_{l}$ does not depend on $\nu$.

THEOREM 3.4. Let

$$
\left\|\partial^{l} u(y, \cdot) / \partial y^{l}\right\|_{L_{p}(-\pi, \pi)} \leq M \quad \text { for any } y>0,
$$

where $l$ is a positive integer. Then the estimates (3.17)-(3.21) are valid, with the norm in (3.17) being that of $L_{p}(-\pi, \pi)$.

The proofs are similar to those of Theorems 3.1 and 3.2.

3.3. Problem C. We can repeat, of course, the method 3.2 for Problem C. This means that when the data are not precisely given, we approximate them by entire functions of exponential type, and therefore all the above results remain valid. But since the data, and so the solution, of Problem $\mathrm{C}$ are periodic, we can approximate the data by trigonometric polynomials.

Let $\tau_{\nu}(t)(\nu=0,1,2, \ldots)$ be a trigonometric polynomial of order not higher than $\nu$, having the following properties:

$$
\begin{aligned}
& \int_{-\pi}^{\pi} \tau_{\nu}(t) d t=1, \\
& \int_{-\pi}^{\pi}\left|\tau_{\nu}(t)\right| d t \leq c \quad(\nu=1,2, \ldots),
\end{aligned}
$$

where $c$ is a constant not depending on $\nu$. Obviously

$$
\tau_{0}(t)=1 /(2 \pi) \text {. }
$$

For $\nu>0$ the polynomials $\tau_{\nu}$ are defined nonuniquely. One may obtain such polynomials, for example, by means of the formula

$$
d_{\nu}(t)=\frac{1}{c_{\nu}}\left(\frac{\sin (\lambda t / 2)}{\sin (t / 2)}\right)^{2 \sigma}
$$


where $\sigma$ is a positive integer not depending on $\lambda, \lambda \in \mathbb{N}^{+}$(then $\left.\nu=(\lambda-1) \sigma\right)$. Here

$$
c_{\nu}=\int_{-\pi}^{\pi}\left(\frac{\sin (\lambda t / 2)}{\sin (t / 2)}\right)^{2 \sigma} d t \sim \lambda^{2 \sigma-1}, \quad \lambda=1,2, \ldots
$$

([60], p. 87). Define

$$
K_{\nu}^{*}(t):=\sum_{k=1}^{l} \frac{(-1)^{k-1}\left(\begin{array}{l}
l \\
k
\end{array}\right)}{k} \sum_{s=0}^{k-1} \tau_{\nu}\left(\frac{t+2 s \pi}{k}\right) .
$$

Then $K_{\nu}^{*}(t)$ is a trigonometric polynomial of order not higher than $\nu$. Furthermore, if

$$
\tau_{\nu}(t)=\sum_{\lambda=-\nu}^{\nu} a_{\lambda} e^{i \lambda t} \quad\left(\bar{a}_{\lambda}=\bar{a}_{-\lambda}\right),
$$

then

$$
\sum_{s=0}^{k-1} \tau_{\nu}\left(\frac{t+2 s \pi}{k}\right)=\sum_{\lambda=-\nu}^{\nu} a_{\lambda} \sum_{s=0}^{k-1} e^{i \lambda(t+2 s \pi) / k}
$$

and therefore

$$
K_{\nu}^{*}(t)=\sum_{k=1}^{l} \frac{(-1)^{k-1}\left(\begin{array}{l}
l \\
k
\end{array}\right)}{k} \sum_{s=0}^{k-1}\left[\sum_{\lambda=-\nu}^{\nu} a_{\lambda} \sum_{s=0}^{k-1} e^{i \lambda(t+2 s \pi) / k}\right]=\sum_{\lambda=-\nu}^{\nu} k_{\lambda}^{*} e^{i \lambda t}
$$

Now let

$$
f_{\nu}(x)=\int_{-\pi}^{\pi} K_{\nu}^{*}(\xi) f(x+\xi) d \xi
$$

Then $f_{\nu}(t)$ is a trigonometric polynomial of order not higher than $\nu$. Furthermore, as an analogue to Lemma 1 we have

Lemma $4([60, \S 5.3])$. Suppose $f \in L_{p}(-\pi, \pi)$ and $f$ has a generalized derivative $f^{(m)}$. Furthermore, suppose that the even nonnegative trigonometric polynomials $\tau_{\nu}(t)$ of order $\nu$ satisfy along with the condition (3.30) the further condition

$$
\int_{0}^{\pi} \tau_{\nu}(t) t^{m} d t \leq \frac{a_{m}}{(\nu+1)^{m}}
$$

where the constant $a_{m}$ does not depend on $\nu=0,1,2, \ldots$ Then the function $f_{\nu}(t)$ defined by (3.39) approximates $f$ in the metric of $L_{p}(-\pi, \pi)$ with the following estimate:

$$
\left\|f-f_{\nu}\right\|_{L_{p}(-\pi, \pi)} \leq b_{m} \frac{\omega_{p}^{k}\left(f^{(m)}, \frac{\pi}{\nu+1}\right)}{(\nu+1)^{m}} \quad \text { for } \nu=0,1,2, \ldots,
$$

where $b_{m}=2\left(\pi^{m}+2 a_{l} / \pi\right), k=l-m$. 
R e m a r k. The function $d_{\nu}(t)$ in (3.33) with $2 \sigma-m \geq 3$ satisfies the condition of Lemma 4.

Now, suppose that instead of the exact $f$ and $g$ in (1.7), we only have the measured $f^{\varepsilon}$ and $g^{\varepsilon}$ such that

$$
\begin{gathered}
\left\|f-f^{\varepsilon}\right\|_{L_{p}(-\pi, \pi)} \leq \varepsilon, \quad\left\|g-g^{\varepsilon}\right\|_{L_{p}(-\pi, \pi)} \leq \varepsilon, \\
f^{\varepsilon}(-\pi)=f^{\varepsilon}(\pi)=g^{\varepsilon}(-\pi)=g^{\varepsilon}(\pi)=0 .
\end{gathered}
$$

For a positive integer $\nu$, let

$$
\begin{aligned}
& f_{\nu}^{\varepsilon}(x)=\int_{-\pi}^{\pi} K_{\nu}^{*} f^{\varepsilon}(x+\xi) d \xi \\
& g_{\nu}^{\varepsilon}(x)=\int_{-\pi}^{\pi} K_{\nu}^{*} g^{\varepsilon}(x+\xi) d \xi .
\end{aligned}
$$

It is not hard to see that

$$
u_{\nu}^{\varepsilon}(y, x)=\sum_{n=0}^{\infty} \frac{(-1)^{n} y^{2 n}\left(f_{\nu}^{\varepsilon}\right)^{(2 n)}(x)}{(2 n) !}+\sum_{n=0}^{\infty} \frac{(-1)^{n-1} y^{2 n-1}\left(g_{\nu}^{\varepsilon}\right)^{(2 n-1)}(x)}{(2 n-1) !}
$$

is the unique solution of Problem $\mathrm{C}$ with the data $f_{\nu}^{\varepsilon}$ and $g_{\nu}^{\varepsilon}$ instead of $f$ and $g$, respectively. On the other hand, from (3.38) and (3.44), (3.45) we have

$$
f_{\nu}^{\varepsilon}(x)=\sum_{\lambda=-\nu}^{\nu}\left(f_{\nu}^{\varepsilon}\right)_{\lambda} e^{i \lambda x}, \quad g_{\nu}^{\varepsilon}(x)=\sum_{\lambda=-\nu}^{\nu}\left(g_{\nu}^{\varepsilon}\right)_{\lambda} e^{i \lambda x},
$$

where

$$
\begin{aligned}
& \left(f_{\nu}^{\varepsilon}\right)_{\lambda}=k_{-\lambda}^{*} \int_{-\pi}^{\pi} f^{\varepsilon}(x) e^{-i \lambda x} d x \\
& \left(g_{\nu}^{\varepsilon}\right)_{\lambda}=k_{-\lambda}^{*} \int_{-\pi}^{\pi} g^{\varepsilon}(x) e^{-i \lambda x} d x .
\end{aligned}
$$

Thus,

$$
u_{\nu}^{\varepsilon}(y, x)=\sum_{\lambda=-\nu}^{\nu}\left(f_{\nu}^{\varepsilon}\right)_{\lambda} \cosh (\lambda y) e^{i \lambda x}+\sum_{\lambda=-\nu}^{\nu}\left(g_{\nu}^{\varepsilon}\right)_{\lambda} \frac{\sinh (\lambda y)}{\lambda} e^{i \lambda x}
$$

is a trigonometric polynomial of order not higher than $\nu$.

The same estimates as in Theorems 3.1, 3.2 can be established, but we do not write them down here again.

Remark. Although our estimates for the regularizing solutions are not of Hölder continuity type, they are stronger than any logarithmic continuity. An important feature is that our estimates are uniform for $y$ with $0<y \leq Y$. This has not been established in earlier works (see, e.g, [38], [43], [46], ...). In [6] Cannon and DuChateau have given a direct process for solving Problem B. 
Their estimates are also not of Hölder continuity type, but are stronger than any logarithmic continuity. They are not valid for all $y \in(0, Y]$, as ours are, but only for $y \in(0, Y / 3]$.

\section{References}

[1] N. A. Aronszajn, A unique continuation theorem for solutions of elliptic partial differential equations or inequalities of second order, J. Math. Pures Appl. 36 (1957), 235-249.

[2] M. A. Atakhodzhaev and U. E. Kobilov, The Cauchy problem for the Laplace equation in an infinite three-dimensional layer, in: Direct and Inverse Problems for Partial Differential Equations and their Applications 186, Fan, Tashkent 1978, $62-75$ (in Russian).

[3] J. Baumeister, Stable Solution of Inverse Problems, Vieweg \& Sohn, Braunschweig 1987.

[4] P. S. Bondarenko and A. V. Rilov, The estimation of the modulus of continuity of the inverse Laplace transform in the solution of the Cauchy problem for Laplace's equation, in: Approximate and Qualitative Methods of the Theory of Differential Equations, Inst. Mat. Akad. Nauk Ukrain. SSR, Kiev 1971, 167-174 (in Russian).

[5] A. P. Calderón, Uniqueness in the Cauchy problem for partial differential equations, Amer. J. Math. 80 (1958), 16-36.

[6] J. R. Cannon and P. DuChateau, Approximating the solution to the Cauchy problem for Laplace's equation, SIAM J. Numer. Anal. 14 (1977), 473-483.

[7] J. R. Cannon and J. Douglas, Jr., The approximation of harmonic and parabolic functions on half-spaces from interior data, in: Numerical Analysis of Partial Differential Equations (C.I.M.E. $2^{0}$ Ciclo, Ispra 1967), Edizioni Cremonese, Rome 1968, 193-230.

[8] J. R. Cannon and K. Miller, Some problems in numerical analytic continuation, SIAM J. Numer. Anal. 2 (1965), 87-98.

[9] T. Carleman, Sur un problème d'unicité pour les systèmes d'équations aux dérivées partielles à deux variables indépendantes, Ark. Mat. Astr. Fys. B 26 (1939), 1-9.

[10] L. A. Chudov, Difference methods for solving Cauchy problem for Laplace's equation, Dokl. Akad. Nauk SSSR 143 (1962), 789-801; English transl.: Soviet Math. Dokl. 3 (1962), 499-503.

[11] D. L. Colton, Improperly posed initial value problem for self-adjoint hyperbolic and elliptic equations, SIAM J. Math. Anal. 4 (1973), 42-51.

[12] D. L. Colton, Partial Differential Equations in the Complex Domain, Pitman, London 1976.

[13] J. Conlan and R. P. Gilbert, Non-linear initial data for second and higher order semilinear elliptic equations, J. Reine Angew. Math. 276 (1975), 1-14.

[14] J. Conlan and G. N. Trytten, Pointwise bounds in the Cauchy problem for elliptic systems of partial differential equations, Arch. Rational Mech. Anal. 22 (1966), 143-152.

[15] H. O. Cordes, Über die Bestimmheit der Lösungen elliptischer Differentialgleichungen durch Anfangsvorgaben, Nachr. Akad. Wiss. Göttingen Math.-Phys. IIa 11 (1956), 239 258

[16] Dinh Nho Hào and R. Gorenflo, A noncharacteristic Cauchy problem for the heat equation, Acta Appl. Math. 24 (1991), 1-27. (See also: Din h Nho Hào and R. Gor enflo, An ill-posed problem for the heat equation, Z. Angew. Math. Mech. 71 (1991), T763T766).

[17] A. Douglis, Uniqueness in Cauchy problems for elliptic systems of equations, Comm. Pure Appl. Math. 13 (1960), 593-608. 
[18] Yu. A. Dubinskiǔ, The algebra of pseudodifferential operators with analytic symbols and its applications to mathematical physics, Uspekhi Mat. Nauk 37 (1982), 97-137; English transl.: Russian Math. Surveys 37 (1982), 107-153.

[19] G. A. Dzhafarli, A uniqueness theorem for the solutions of an elliptic system in the half-plane, Dokl. Akad. Nauk Azerbaidzhan. SSR 26 (1970), 12-14 (in Russian).

[20] Kh. Sh. Dzhuraev, On a solution of the Cauchy problem for the Laplace equation, Dokl. Akad. Nauk Tadzhik. SSR 29 (1986), 506-509 (in Russian).

[21] R. S. Falk and P. B. Monk, Logarithmic convexity for discrete harmonic functions and the approximation of the Cauchy problem for the Poisson's equation, Math. Comp. 47 (1986), 135-149.

[22] Yu. L. Gapanenko, Stability of the solution of the Cauchy problem for the Laplace equation on a weak compactum, in: Numerical Mathematics and Sofware, Moskov. Gos. Univ., 1985, 44-50 (in Russian).

[23] V. I. Gorbar̆chuk, Estimates of the accuracy of an approximate solution for the Cauchy problem for the Laplace equation in an infinite strip, Ukrain. Mat. Zh. 32 (1980), 731-736; English transl.: Ukrainian Math. J. 32 (1980), 489-494.

[24] R. Gorenflo, Funktionentheoretische Bestimmung des Aussenfeldes zu einer zweidimensionalen magnetohydrostatischen Konfiguration, Z. Angew. Math. Phys. 16 (1965), 279290.

[25] —, Behandlung ebener magnetohydrostatischer Gleichgewichtsprobleme mittels komplexer Analysis, in: Tagung Freie Randwertaufgaben, E. Grafarend und N. Weck (eds.), Mitteilungen aus dem Institut für Theoretische Geodäsie der Universität Bonn, N.4, 1972, $2-18$.

[26] J. Hadamard, Sur les problèmes aux derivées partielles et leur signification physique, Bull. Univ. Princeton 13 (1902), 49-32.

[27] - Lectures on Cauchy's Problem in Linear Partial Differential Equations, Yale Univ. Press, 1923.

[28] H. D. Han, The finite element method in the family of improperly posed problems, Math. Comp. 38 (1982), 55-65.

[29] E. Heinz, Über die Eindeutigkeit beim Cauchyschen Anfangswertproblem einer elliptischen Differentialgleichung zweiter Ordnung, Nachr. Akad. Wiss. Göttingen Math.-Phys. IIa 1 (1955), 1-12.

[30] L. Hörmander, Linear Partial Differential Operators, Springer, Berlin 1976.

[31] —, Uniqueness theorems for second order elliptic differential equations, Comm. Partial Differential Equations 8 (1983), 21-64.

[32] V. K. Ivanov, The Cauchy problem for the Laplace equation in an infinite strip, Differentsial'nye Uravneniya 1 (1965), 131-136 (in Russian).

[33] F. John, A note on "improper" problems in partial differential equations, Comm. Pure Appl. Math. 8 (1955), 494-495.

[34] -, Differential Equations with Approximate and Improper Data, New York University, 1955.

[35] -, Continuous dependence on the data for solutions of partial differential equations with a predescribed bound, Comm. Pure Appl. 13 (1960), 551-586.

[36] L. A. Knizhnerman, Numerical solution of the Cauchy problem for the Laplace equation by means of an expansion in Fourier-Chebyshev series, Izv. Akad. Nauk SSSR Ser. Fiz. Zemli 1984 (10), 76-81 (in Russian).

[37] R. J. Knops and L. E. Payne, Uniqueness and continuous dependence of the null solution in the Cauchy problem for a nonlinear elliptic system, in: Inverse and Improperly Posed Problems in Differential Equations, G. Anger (ed.), Akademie-Verlag, Berlin 1979, 151-160. 
[38] T. I. Korolyuk, The Cauchy problem for the Laplace equation, Izv. Vyssh. Uchebn. Zaved. Mat. 130 (1973), 53-55 (in Russian).

[39] H. Kumano-go, On the uniqueness of the solution of the Cauchy problem and the unique continuation theorem for elliptic equations, Osaka Math. J. 14 (1962), 182-212.

[40] E. M. Landis, Certain properties of equations of elliptic type, Dokl. Akad. Nauk SSSR 107 (1956), 640-643 (in Russian).

[41] - Some questions in the qualitative theory of elliptic and parabolic equations, Uspekhi Mat. Nauk 14 (1) (1959), 21-85; English transl. in Amer. Math. Soc. Transl. (2) 20 (1962), 173-238.

[42] - Some questions in the qualitative theory of second-order elliptic equations (case of several independent variables), Uspekhi Mat. Nauk 18 (1) (1963), 3-62; English transl. in Russian Math. Surveys 18 (1963), 1-62.

[43] M. M. Lavrent'ev, On the Cauchy problem for the Laplace equation, Izv. Akad. Nauk SSSR Ser. Mat. 120 (1956), 819-842 (in Russian).

[44] - On the Cauchy problem for linear elliptic equations of second order, Dokl. Akad. Nauk SSSR 112 (1957), 195-197 (in Russian).

[45] -, Some Improperly Posed Problems in Mathematical Physics, Springer, New York 1967.

[46] M. M. Lavrent'ev, V. G. Romanov and S. P. Shishat-skiŭ, Ill-posed Problems of Mathematical Physics and Analysis, Transl. Math. Monographs, Amer. Math. Soc., Providence, R.I., 1986.

[47] N. Lerner, Unicité du problème de Cauchy pour des opérateurs elliptiques, Ann. Sci. École Norm. Sup. (4) 17 (1984), 469-505.

[48] H. A. Levine and S. Vessella, Estimates and regularization for solutions of some ill-posed problems of elliptic and parabolic type, Rend. Circ. Mat. Palermo 123 (1986), 161-183.

[49] B. H. Li and Y. Q. Li, On the initial value problem of the Laplace equation, J. Systems Sci. Math. Sci. 7 (1987), 1-6.

[50] O. A. Liskovets, A solution of the Cauchy problem for the Laplace equation by a generalized method of the summability of series, Vestsi Akad. Navuk BSSR Ser. Fiz.-Mat. Navuk 1970 (4), 68-74 (in Russian).

[51] V. G. Mazya and V. P. Khavin, On the solutions of the Cauchy problem for Laplace's equation (solvability, normality, approximation), Trans. Moscow Math. Soc. 30 (1974), $65-117$.

[52] L. A. Medeiros, Remarks on a non well-posed problem, Proc. Roy. Soc. Edinburgh 102A (1986), 131-140.

[53] S. M. Mergelyan, Harmonic approximation and approximate solution of the Cauchy problem for the Laplace equation, Uspekhi Mat. Nauk 11 (1956), 3-26 (in Russian).

[54] F. Meyer und H. U. Schmidt, Torusartige Plasmakonfigurationen ohne Gesamtstrom durch ihren Querschnitt im Gleichgewicht mit einem Magnetfeld, Z. Naturforsch. 13a (1958), 1005-1015.

[55] K. Miller, Three circle theorems in partial differential equations and applications to improperly posed problems, Arch. Rational Mech. Anal. 16 (1964), 126-154.

[56] - Least squares methods for ill-posed problems with a prescribed bound, SIAM J. Math. Anal. 1 (1970), 52-74.

[57] S. Mizohata, Unicité dans les problèmes de Cauchy pour quelques équations différentielles elliptiques, Mem. Coll. Sci. Univ. Kyoto Ser. A Math. 31 (1958), 121-128.

[58] -, Unicité du prolongement des solutions des équations elliptiques du quatrième ordre, Proc. Japan Acad. 34 (1958), 687-692.

[59] C. Müller, On the behavior of the solutions of the differential equation $\Delta u=F(x, u)$ in the neighborhood of a point, Comm. Pure Appl. Math. 7 (1954), 505-514. 
[60] S. M. Nikol'skiǔ, Approximation of Functions of Several Variables and Imbedding Theorems, Springer, Berlin 1975 (translated from the Russian).

[61] L. Nirenberg, Uniqueness in Cauchy problems for differential equations with constant leading coefficients, Comm. Pure Appl. Math. 10 (1957), 89-105.

[62] L. E. Payne, Bounds in the Cauchy problem for the Laplace equation, Arch. Rational Mech. Anal. 5 (1960), 35-45.

[63] - On a priori bounds in the Cauchy problem for elliptic equations, SIAM J. Math. Anal. 1 (1970), 82-89.

[64] -, Improperly Posed Problems in Partial Differential Equations, SIAM, Philadelphia, Penn., 1975.

[65] L. E. Payne and D. Sather, On some improperly posed problems for quasilinear equations of mixed type, Trans. Amer. Math. Soc. 128 (1967), 135-141.

[66] - - - On some improperly posed problems for the Chaplygin equation, J. Math. Anal. Appl. 19 (1967), 67-77.

[67] - - - On an initial-boundary value problem for a class of degenerate elliptic operators, Ann. Mat. Pura Appl. 78 (1968), 323-337.

[68] A. P. Poddubnyak and V. E. Emets, The Cauchy problem for the Laplace equation in an infinite $n+1$-dimensional layer, Mat. Metody i Fiz.-Mekh. Polya 15 (1982), 13-15 (in Russian).

[69] M. H. Protter, Unique continuation for elliptic equations, Trans. Amer. Math. Soc. 95 (1960), 81-91.

[70] C. Pucci, Sui problemi di Cauchy non "ben posti", Rend. Accad. Naz. Lincei 8 (18) (1955), 473-477.

[71] —, Discussione del problema di Cauchy per le equazioni di tipo ellittico, Ann. Mat. Pura Appl. 46 (1958), 131-153.

[72] - Some Topics in Parabolic and Elliptic Equations, Lecture Series No. 36, Institute for Fluid Mechanics and Applied Mathematics, University of Maryland, 1958.

[73] A. Romanovich and A. V. Fursikov, On the question of the solvability of the Cauchy problem for the Laplace operator, Vestnik Moskov. Gos. Univ. Ser. I Mat. Mekh. 1987 (3), $78-80$

[74] J.-C. Saut et B. Scheurer, Sur l'unicité du problème de Cauchy et le prolongement unique pour des équations elliptiques à coefficients non localement bornés, J. Differential Equations 43 (1982), 28-43.

[75] -, - Unique combination and uniqueness of the Cauchy problem for elliptic equations with unbounded coefficients, in: Nonlinear Partial Differential Equations and their Applications, Collège de France Séminaire, Vol. V (Paris 1981/ 1982), Res. Notes in Math. 93, Pitman, Boston, Mass., 1983, 260-275.

[76] P. W. Schaefer, On the Cauchy problem for an elliptic system, Arch. Rational Mech. Anal. 20 (1965), 391-412.

[77] P. W. Schaefer, Pointwise bounds in the Cauchy problem for an elliptic system, SIAM J. Appl. Math. 15 (1967), 665-677.

[78] - Improvable estimates in some non-well-posed problems for a system of elliptic equations, SIAM J. Math. Anal. 4 (1973), 447-455.

[79] - On uniqueness, stability, and pointwise estimates in the Cauchy problem for coupled elliptic equations, Quart. Appl. Math. 31 (1973), 321-328.

[80] S. D. Shalaginov, The Cauchy problem for the Laplace equation in a complex space, Differentsial'nye Uravneniya 16 (1980), 947-949 (in Russian).

[81] A. N. Tikhonov and V. Y. Arsenin, Solution of Ill-posed Problems, Wiley, New York 1977 (Russian third ed., 1986).

[82] N. V. Tkachenko, Solution of a certain Cauchy problem for the Laplace equation, in: Math. Physics, Leningrad Gos. Ped. Inst., Leningrad 1987, 27-32 (in Russian). 
[83] G. Trytten, Pointwise bounds for solutions of the Cauchy problem for elliptic equations, Arch. Rational Mech. Anal. 13 (1963), 222-244.

[84] Tran Duc Van, On the pseudodifferential operators with real analytic symbols and their applications, J. Fac. Sci. Univ. Tokyo Sect. IA Math. 36 (1989), 803-825.

[85] Tran Duc Van, Dinh Nho Hào and R. Gorenflo, Approximating the solution to the Cauchy problem and the boundary problem for the Laplace equation, to appear.

[86] Tran Duc Van, Dinh Nho Hào, Trinh Ngoc Minh and R. Gorenflo, On the Cauchy problems for systems of partial differential equations with a distinguished variable, Numer. Funct. Anal. Optim. 12 (1\&2) (1991), 213-236.

[87] Tran Duc Van, Nguyen Duy Thai Son and Dinh Zung, Approximately solving Cauchy problem for the wave equation by the method of differential operators of infinite order, Acta Math. Vietnam. 13 (2) (1988), 127-136.

[88] Trinh Ngoc Minh and Tran Duc Van, Cauchy problems for systems of partial differential equations with a distinguished variable, Dokl. Akad. Nauk SSSR 284 (1985), 507-510; English transl.: Soviet Math. Dokl. 32 (1985), 562-565.

[89] Trinh Ngoc Minh, Linear differential operators of infinite order and their applications, Acta Math. Vietnam. 12 (1) (1987) 101-124.

[90] P. N. Vabishchevich, On the solution of the Cauchy problem for the Laplace equations in a doubly connected domain, Dokl. Akad. Nauk SSSR 241 (1978), 1257-1260; English transl.: Soviet Math. Dokl. 19 (1978), 976-980.

[91] - Numerical solution of the Cauchy problem for elliptic equations and systems, Vestnik Moskov. Univ. Ser. XV Vychisl. Mat. Kibernet. 1979 (3), 3-10 (in Russian).

[92] P. N. Vabishchevich, V. B. Glasko and Yu. A. Kriksin, Solution of the Hadamard problem by a Tikhonov-regularizing algorithm, U.S.S.R. Comput. Math. and Math. Phys. 19 (1979), 103-112.

[93] P. N. Vabishchevich and P. A. Pulatov, A method of numerical solution of the Cauchy problem for elliptic equations, Vestnik Moskov. Gos. Univ. Ser. XV Vychisl. Mat. Kibernet. 1984, 3-8.

[94] K. Watanabe, On the uniqueness of the Cauchy problem for certain elliptic equations with triple characteristics, Tôhoku Math. J. (2) 23 (1971), 473-490.

[95] —, A unique continuation theorem for an elliptic operator of two independent variables with nonsmooth double characteristics, Osaka J. Math. 10 (1973), 243-246.

[96] _, Remarque sur l'unicité dans les problèmes de Cauchy pour les opérateurs différentiels elliptiques à caractéristiques de multiplicité constante et au plus triple, C. R. Acad. Sci. Paris Sér. A-B 284 (1977), A1385-A1386.

[97] K. Watanabe et C. Zuily, Unicité du problème de Cauchy pour une classe d'opérateurs différentiels elliptiques à caractéristiques de multiplicité variable, C. R. Acad. Sci. Paris Sér. A-B 283 (1976), A627-A630.

[98] —, -, Unicité du problème de Cauchy pour une classe d'opérateurs différentiels elliptiques à caractéristiques de multiplicité variable, Sém. Goulaouic-Schwartz 1976/1977, Équations aux dérivées partielles et analyse fonctionnelle, Exp. No. 15, 9 pp., Centre Math., École Polytech., Palaiseau 1977.

[99] - - - On the uniqueness of the Cauchy problem for elliptic differential operators with smooth charateristics of variable multiplicity, Comm. Partial Differential Equations 2 (1977), 831-855.

[100] A. Yanushauskas, A Cauchy problem for Laplace's equation and the multiplication operation for harmonic functions, Dokl. Akad. Nauk SSSR 159 (1964), 286-289 (in Russian).

[101] - Entire solutions of second order elliptic equations, Siberian Math. J. 11 (1970), 863869. 
[102] A. Yanushauskas, On the Cauchy problem for the Laplace equation with three independent variables, ibid. 16 (1975), 1040-1047.

[103] Sh. Yarmukhamedov, On the Cauchy problem for Laplace's equation, Dokl. Akad. Nauk SSSR 235 (1977), 281-283, English transl.: Soviet Math. Dokl. 18 (1977), 939-882.

[104] - , The Cauchy problem for the Laplace equation in an infinite domain, Dokl. Akad. Nauk UzSSR 1980 (12), 9-10 (in Russian).

[105] _, The Cauchy problem for the Laplace equation in M. M. Lavrent'ev's formulation, in: Ill-posed Problems of Mathematical Physics and Analysis, A. S. Alekseev (ed.), Nauka, Sibirsk. Otdel., Novosibirsk 1984, 203-209 (in Russian).

[106] C. Zuily, Unicité du problème de Cauchy pour des opérateurs elliptiques à caractéristiques de hautes multiplicités, Comm. Partial Differential Equations 10 (1985), 219-244.

Added in proof. Recently, there have been some new papers on the Cauchy problem for elliptic equations:

1. Dinh Nho Hào, A mollification method for ill-posed problems, preprint A-92-35, FB Mathematik, FU Berlin.

2. A. V. Fursikov, The Cauchy problem for a second-order elliptic equation in a conditionally well-posed formulation, Trans. Moscow Math. Soc. 52 (1990), 139-176.

3. M. V. Klibanov and F. Santosa, A computational quasi-reversibility method for Cauchy problems for Laplace's equation, SIAM J. Appl. Math. 5 (1991), 1653-1675. 